Discussion Paper No. 857

\title{
ASKING ONE TOO MANY? \\ WHY LEADERS NEED TO BE DECISIVE
}

\author{
Junichiro Ishida \\ Takashi Shimizu
}

October 2012

The Institute of Social and Economic Research

Osaka University

6-1 Mihogaoka, Ibaraki, Osaka 567-0047, Japan 


\title{
Asking One Too Many? Why Leaders Need to Be Decisive*
}

\author{
Junichiro Ishida ${ }^{\dagger}$ and Takashi Shimizu ${ }^{\ddagger}$
}

October 23, 2012

\begin{abstract}
It is often touted that decisiveness is one of the most important qualities to be possessed by leaders, broadly defined. To see how and why decisiveness can be a valuable asset in organizations, we construct a model of strategic information transmission where: (i) a decision maker solicits opinions sequentially from experts; (ii) how many experts to solicit opinions from is the decision maker's endogenous choice. We show that communication is less efficient when the decision maker is indecisive and cannot resist the temptation to ask for a second opinion. This result suggests that the optimal diversity of information sources depends critically on the strategic nature of communication: when communication is strategic, it is optimal to delegate information acquisition to a single party and rely exclusively on it; when it is not, it is optimal to diversify information sources and aggregate them via communication.
\end{abstract}

Keywords: Decisiveness, Cheap talk, Multiple senders, Commitment.

JEL Classification Number: D23; D82.

\footnotetext{
${ }^{*}$ The second author was financially supported by Kansai University's Overseas Research Program for the year of 2009. Of course, any remaining errors are our own.

${ }^{\dagger}$ Institute of Social and Economic Research, Osaka University, 6-1 Mihogaoka, Ibaraki-shi, Osaka 567-0047 JAPAN (E-mail: jishida@iser.osaka-u.ac.jp)

${ }^{\ddagger}$ Faculty of Economics, Kansai University, 3-3-35 Yamate-cho, Suita-shi, Osaka 564-8680 JAPAN (E-mail: tshimizu@ipcku.kansai-u.ac.jp)
} 
"If I had to sum up in a word what makes a good manager, I'd say decisiveness."

- Lee Iacocca

"In any moment of decision, the best thing you can do is the right thing, the next best thing is the wrong thing, and the worst thing you can do is nothing."

- Theodore Roosevelt

\section{Introduction}

It is often touted that decisiveness is one of the most important qualities to be possessed by leaders. However, while this type of assertion is intuitively appealing and appears to be well received within the business community, it is not exactly clear how the leader's decisiveness works to improve the organization efficiency in any way. In this paper, we construct a simple model of strategic information transmission with multiple senders, which falls into a class of cheap-talk models, to explore into this issue. Using the model, we illustrate how the presence of a decisive leader keeps the followers' incentives straight and mitigates opportunistic strategic behaviors by them.

The model we consider is as follows. There are a decision maker (female) and two experts (male), each of whom has his own private information. As always in this type of model, the preferences of the decision maker and those of each expert are not perfectly aligned, making truthful communication an issue to be reckoned with. The key departure, which distinguishes our setup from the existing models of cheap talk with multiple senders, is that it is the decision maker's discretion how many experts to solicit opinions from. In this setting, we ask whether there are such things as "asking too many opinions," i.e., a situation in which the decision maker cannot resist the temptation to ask for a second opinion even though she would be better off making up her mind after soliciting one opinion and immediately initiating an action. We say that the decision maker is indecisive when she cannot resist the temptation to ask for a second opinion.

The current framework shows that there is indeed a case where the decision maker's indecisiveness proves to be costly for the organization as a whole, as it obstructs efficient information transmission by making each expert's problem highly strategic in nature. The basic logic behind this result stems from an insight recently developed in the analyses of cheap-talk models with an imperfectly informed receiver or multiple imperfectly informed senders (Chen [4], Lai [14], Moreno de Barreda [6], Ishida and Shimizu [13] for models with an informed receiver; Austen-Smith [2], Morgan and Stocken [15], Galeotti et al. [11] for models 
with multiple senders). ${ }^{1}$ In this growing body of literature, it is found that under a wide range of circumstances, the more informed the receiver becomes, the less information she can extract from the sender. This implies that the incentive for each of the experts to communicate truthfully diminishes when he knows that the decision maker will solicit opinions from an alternative source and becomes "more informed" (from the expert's viewpoint). Building on this logic, we show that there exists no truth-telling equilibrium when the decision maker is known to be indecisive and cannot resist the temptation to ask for a second opinion, meaning that information cannot be aggregated efficiently under the lead of an indecisive leader. This also means that the decision maker may be made better off by committing herself to the "one-and-done" strategy, i.e., soliciting only one opinion and initiating an action right away, if such an option is ever feasible.

As one of the main contentions, our analysis provides an insight into the optimal diversity of information sources within an organization. To see this, consider an environment where the decision maker has some discretion over the distribution of information, so that the number of information sources is her endogenous choice. Intuitively, there is a gain from diversifying information sources and obtaining information from many different angles, as it allows the decision maker to aggregate different sources of information to make a better estimate of the current state. The argument indeed holds true when the underlying environment is nonstrategic, and information can be solicited via communication at no cost: in this case, it is optimal to diversify information sources to complement each other and aggregate them via communication. This argument does not necessarily apply, though, when there is a potential conflict of interests between the decision maker and the experts, and communication is more strategic in nature. Our analysis suggests that having multiple information sources can be an impediment to efficient information transmission and actually lower the decision maker's welfare. In strategic environments, therefore, it is optimal to delegate information acquisition to a single party and rely on it exclusively.

While the importance of leadership has been widely recognized in various fields, economists have paid relatively less attention to this issue until recently. There is now a growing body of literature which explores the nature of leadership in formal economic analyses; most of the existing studies largely focus on the informational aspect of leadership, as giving a sense of direction or a focal point to coordinate beliefs and behaviors. One of the early attempts is made by Hermalin [12] who formalizes the notion of leadership in the context of "leading by example" where a leader transmits private information by moving

\footnotetext{
${ }^{1}$ There is evidently a close connection between models with an informed receiver and those with multiple receivers. The case with an informed receiver can be regarded as a subgame of models with multiple sender after one of the senders reports truthfully.
} 
first. Rotemberg and Saloner [17] model vision as a bias which makes the manager favor one project over the other. Hiring a manager with a vision can be beneficial because it reduces uncertainty as to what kind of employee initiatives will be favored later. ${ }^{2}$ Van den Steen [7] also formalizes the notion of vision and shows that a leader with strong beliefs would attract employees with similar beliefs. This sorting effect gives direction to the firm and improves coordination, suggesting a channel through which strong vision can affect the firm's performance. Dewan and Myatt [8] [9] [10] and Bolton et al. [3] explore the role of leadership in the beauty-contest type setting where adaptation and coordination are the main concern. The current work differs from the existing literature in that our explicit focus is on the role of the leader's "decisiveness" and its impact on the quality of communication in strategic environments.

We illustrate our main contention by building on and extending a model of strategic information transmission developed in Ishida and Shimizu [13]. ${ }^{3}$ The paper proceeds as follows. In section 2, we outline the basic framework. In section 3, we analyze two extreme cases to show how the decision maker's decisiveness facilitates information transmission in strategic environments. In section 4, we provide some implications of the model. Finally, in section 5, we offer some concluding remarks.

\section{A model of public consultation}

Setup: Consider a two-period model with a decision maker (female) and two experts (male). ${ }^{4}$ The decision maker's task in each period $t=1,2$ is to collect information about the state of nature, either by garnering evidence on her own or by communicating with an expert, and take an appropriate action based on the acquired information. For clarity, we refer to an expert who is called upon to make advice in period $t$ as expert $t$. The (time-invariant) state of nature is denoted by $\theta \in\{0,1\}$, where each state is equally likely ex ante:

$$
\operatorname{Pr}(\theta=0)=\operatorname{Pr}(\theta=1)=0.5
$$

The state of nature is not directly observable to anyone.

\footnotetext{
${ }^{2}$ Similarly, Rotemberg and Saloner [16] argue that a firm could be better off by committing to a specific business strategy.

${ }^{3}$ It is important to note that, although our analytical framework builds on a particular model of strategic information transmission, our logic can be applied to many other cases as long as the base model has a feature that communication becomes less effective when the receiver amasses more information. The class of models with this feature includes many variants of the canonical model of cheap talk such as Morgan and Stocken [15].

${ }^{4}$ Since the decision maker can solicit one opinion in one period, she can ask for at most two opinions. We assume that there are only two periods strictly to keep the analysis as simple as possible while keeping the substance of the model. As we will later argue, this assumption is quite inessential for our main results.
} 
[Remark] Here, we consider the case of public consultation in which each expert knows whether he is the first or second one to make advice. This assumption of public consultation is made mostly for analytical clarity and does not affect our main contention in a qualitative sense: we later extends the analysis to the case of private consultation in which communication is done in private and each expert does not know whether he is the first or second one to make advice.

Information: At the beginning of each period, nature randomly draws a signal $\hat{\theta}_{t} \in\{0,1\}$ which is imperfectly correlated with $\theta$. The conditional distribution of the signal is given by

$$
\operatorname{Pr}\left(\hat{\theta}_{t}=i \mid \theta=i\right)=r, t=1,2, i=0,1,
$$

where $r \in(0.5,1)$ measures the accuracy of the signal. We assume that expert $t$, with enough expertise, can always observe $\hat{\theta}_{t}$. Lacking expertise (or resources to expend) on the problem at hand, however, the decision maker can observe a signal only with some probability: let $p_{t}$ denote the probability that she directly observes $\hat{\theta}_{t} \cdot{ }^{5}$ If the decision maker fails to observe a signal in period $t$, she must then consult expert $t$ to solicit this information via (strategic) communication as we describe below.

Throughout the analysis, we focus on the case where things unfold over time and it becomes more likely for the decision maker to come across useful information by chance: for instance, one can conceive of a situation where uncertainty regarding the state of nature partially resolves due to some exogenous events or the arrival of "news" from some nonstrategic outside sources. Formally, we capture this situation by supposing $p_{1}=0$ and $p_{2}=p>0 .{ }^{6}$ The parameter $p$ is one of the key parameters of the model which measures how difficult it is for the decision maker to obtain unbiased information about $\theta$ and hence how much she needs to rely on the experts to make up her mind.

Communication: When the decision maker fails to observe a signal on her own, she needs to solicit this information via communication. We consider a simple (cheap-talk) message game: in each period $t$, upon observing $\hat{\theta}_{t}$, expert $t$ sends a costless message $m_{t} \in\{0,1\}$ to the decision maker. We assume that expert 2 can observe $m_{1}$, although this assumption turns out to be totally irrelevant. ${ }^{7}$

Action: Given all the available information, the decision maker must take some action $a_{t} \in\{0,1, W\}$ in each period, where $a_{t}=W$ denotes the "wait" option. For expositional

\footnotetext{
${ }^{5}$ If the decision maker happens to observe a signal, the information-acquisition stage ends immediately as no more information is available for the period.

${ }^{6}$ Alternatively, one can regard that we focus on the case where the decision maker fails to observe a signal in period 1 .

${ }^{7}$ As we will see shortly, expert 2 is called upon to make advice only when $m_{1}=1$ and hence effectively knows what message is sent by expert 1 anyway.
} 
purposes, we say that the decision maker takes an action immediately when $a_{1} \neq W$ and that she waits and asks for a second opinion when $a_{1}=W$. The game ends at the end of period 1 if the decision maker chooses to take an action immediately. If she chooses to wait, i.e., $a_{1}=W$, on the other hand, the game proceeds to the next period with an additional cost $d .^{8}$

Preferences: Each player has his or her own preferred action and derives a private benefit from implementing the preferred action. We assume that the decision maker prefers $a=0$ while each expert prefers $a=1$, so that their preferences are not perfectly aligned. The payoff for the decision maker when she takes an action in period $t$, i.e., $a_{t} \neq W$, is given by

$$
u^{d}(\theta, a, t)=\mathbb{I}(\theta=a)+b \mathbb{I}(a=0)-d(t-1),
$$

while that for each expert is

$$
u^{e}(\theta, a)=\mathbb{I}(\theta=a)+b \mathbb{I}(a=1)
$$

where $b>0$ is the parameter which measures the salience of the private benefit. If the decision maker takes no action at all $\left(a_{1}=a_{2}=W\right)$, the payoff is zero for all players.

\section{Analysis}

\subsection{The decision maker's problem}

We first consider the decision maker's problem in period 2. Let $\sigma_{t}=\operatorname{Pr}\left(\theta=1 \mid \Omega_{t}\right)$ denote the decision maker's belief in period $t$, where $\Omega_{t}$ denotes her information set available in period $t$. Given all the available information, the decision maker chooses $a_{2}=W$ (taking no action at all) if

$$
\max \left\{1-\sigma_{2}+b, \sigma_{2}\right\}<0
$$

It follows from this that it is never optimal for the decision maker to choose $a_{2}=W{ }^{9}$

\footnotetext{
${ }^{8}$ To focus on more meaningful cases, we assume throughout the analysis that it is always optimal for the decision maker to solicit at least one opinion. Formally, we circumvent this issue by assuming that the cost of soliciting the first opinion is zero. In general, the model can be applied to any circumstances where it is prohibitively costly for the decision maker to choose an action based solely on her own prior information. For instance, the state of nature may be two-dimensional, consisting of $\theta$ and the other unmodeled state, and the sender sends a message about both of these dimensions. There are sufficiently many states along this unmodeled dimension and at least one of them yields a prohibitively low payoff to both players when the corresponding action is mistakenly chosen, as assumed in Aghion and Tirole (1997). The first opinion is then always desirable to avoid this mistake when its cost is sufficiently large for both players.

${ }^{9}$ We assume throughout the analysis that the decision maker chooses either $a_{t}=0$ or $a_{t}=1$ over $a_{t}=W$ if she is indifferent between the two. Similarly, she always chooses $a_{t}=0$ (her preferred action) over $a_{t}=1$ when she is indifferent.
} 
Given this, the decision maker in period 2 chooses $a_{2}=0$ over $a_{2}=1$ if

$$
1-\sigma_{2}+b \geq \sigma_{2} \Leftrightarrow B:=\frac{1+b}{2} \geq \sigma_{2} .
$$

The expected payoff conditional on the posterior belief, denoted by $x\left(\sigma_{2}\right)$, is then given by

$$
x\left(\sigma_{2}\right)= \begin{cases}\sigma_{2} & \text { if } \sigma_{2}>B, \\ 1-\sigma_{2}+b & \text { if } B \geq \sigma_{2} .\end{cases}
$$

The decision maker's problem in period 1 is more complicated because the problem is now dynamic in nature. First, it is clear from the argument above that one can at least rule out $a_{1}=1$ if $B \geq \sigma_{1}$ and $a_{1}=0$ if $\sigma_{1}>B$. For $B \geq \sigma_{1}$, the receiver chooses $a_{1}=0$ over $a_{1}=W$ if

$$
1-\sigma_{1}+b \geq E\left(x\left(\sigma_{2}\right) \mid \sigma_{1}\right)-d .
$$

For $\sigma_{1}>B$, the receiver chooses $a_{1}=1$ over $a_{1}=W$ if

$$
\sigma_{1} \geq E\left(x\left(\sigma_{2}\right) \mid \sigma_{1}\right)-d .
$$

We seek for a PBE in which communication matters. ${ }^{10}$ Since the model admits a plethora of equilibria, many of which are not particularly illuminating for the purpose of the current analysis, we focus the following parameter space to restrict our attention to more relevant cases.

Assumption $1 r>\frac{1}{2}+b$.

The assumption assures that each expert's information is accurate enough to be valuable to the decision maker by itself. What this assumption means is as follows. Suppose that expert 1 observes $\hat{\theta}_{1}=1$ and recommends $m_{1}=1$. If the decision maker follows the advice, the expected payoff is $r$. If she does not rely on the expert's information and chooses $a_{1}=0$, on the other hand, the expected payoff is $1 / 2+b$. The assumption thus states that the decision maker can be made better off with the expert's advice, even in the worst case for her, than without it. Note also that the assumption implies $r>B$.

Within this environment, we say that there exists a fully separating truth-telling equilibrium if all of the experts, who are called upon to make advice on the equilibrium path, adopt the truth-telling strategy, i.e., $m_{t}=\hat{\theta}_{t}$. The following two forms are feasible candidates for our notion of truth-telling equilibrium:

\footnotetext{
${ }^{10} \mathrm{As}$ in prototype cheap-talk models, there always exists a babbling equilibrium in each stage where the expert lies and the decision maker simply ignores the message. For any parameter values, therefore, there exists an uninformative equilibrium where both experts always lie and the decision maker always chooses $a_{1}=0$ regardless of $m_{1}$. Here, we disregard this possibility and seek for equilibria where at least one of the experts communicates truthfully with positive probability on the equilibrium path.
} 
- The decision maker always takes an action immediately, and expert 1 adopts the truthtelling strategy (regardless of expert 2's strategy off the equilibrium path);

- The decision maker asks for a second opinion with some positive probability, and both of the experts adopt the truth-telling strategy.

\subsection{Communication when the receiver is decisive}

We first consider the case where the cost of waiting, captured by $d$, is relatively high and the decision maker takes an action immediately after one period. This greatly simplifies the analysis because expert 1 knows that he is the only one to make advise on the equilibrium path. The problem is thus effectively reduced to a simple static one.

Under Assumption 1, the decision maker simply takes an action recommended by the expert, provided that he adopts the truth-telling strategy. The expert truthfully reveals his private information if

$$
r \geq 1-r+b \Leftrightarrow r \geq B
$$

which also holds by Assumption 1.

This means that under the maintained assumption that each expert's information is sufficiently accurate, there is always a truth-telling equilibrium as long as the decision maker takes an action immediately after one period. The question then eventually comes down to whether the decision maker is able to do so in a credible manner. To this end, there are two cases we need to consider, depending on the first message $m_{1}$. Suppose first that the decision maker deviates and asks for a second opinion when $m_{1}=0$. In this case, even if expert 2 also communicates truthfully, the decision maker always chooses $a_{2}=0$ regardless of $m_{2}$. This means that the decision maker has no incentive to deviate because she cannot gain any useful information in period 2.

The decision maker thus has an incentive to deviate only when $m_{1}=1$ : in this case, she may change her choice of action, depending on the information she obtains in period 2 . Now suppose that she deviates and asks for a second opinion. One can then show that the incentive compatibility constraint for expert 2 cannot be satisfied; this fact is the driving force of the model, and we will explore this point in greater detail in the next subsection (and also in the proof of Proposition 1). The decision maker can thus gain useful information only if she can gain useful information on her own, which occurs with probability $p$. If this information is not valuable enough, the decision maker takes an action immediately, thereby making full separation feasible. 
Proposition 1 There exists a fully separating truth-telling equilibrium if $d \geq 2 \operatorname{pr}(1-r) b$. In equilibrium, the decision maker adopts the one-and-done strategy, i.e., always takes an action immediately after soliciting an opinion from expert 1.

Proof: See Appendix.

\subsection{Communication when the receiver is indecisive}

The decision maker is tempted to ask for a second opinion only when $m_{1}=1$, i.e., the first news is not favorable to her. When $d$ goes below the threshold provided in Proposition 1, she may start asking for a second opinion upon receiving a bad news. The nature of the game then alters substantially, now that there are two experts to communicate with the decision maker.

The first question to be asked is whether there could exist a fully separating equilibrium when the decision maker asks for a second opinion. To see this, suppose that expert 2 adopts the truth-telling strategy and investigate expert 1's incentives. If expert 1 also communicates truthfully when $\hat{\theta}_{1}=0$, the decision maker takes an action immediately and the expected payoff is $r$. If the expert deviates and claims $m_{1}=1$, on the other hand, the decision maker will ask for a second opinion. If $m_{2}=0$ (which occurs with probability $r^{2}+(1-r)^{2}$, given that expert 2 communicates truthfully), the decision maker chooses $a_{2}=0$ which is correct with probability $r^{2} /\left[r^{2}+(1-r)^{2}\right]$; if $m_{2}=1$ (which occurs with probability $2 r(1-r)$ ), she chooses $a_{2}=1$ which is correct with probability $1 / 2$. Given this argument, the condition for truth telling (for expert 1 ) is obtained as

$$
r \geq r^{2}+r(1-r)+2 r(1-r) b=r+2 r(1-r) b,
$$

which is clearly never satisfied as long as $b>0$, no matter how small it is. This means that there exists no truth-telling equilibrium when the decision maker cannot resist the temptation to ask for a second opinion, thereby illuminating a virtue of being decisive in strategic environments.

Intuitively, the driving force of this result is the diversification of responsibility: when there are multiple exerts to make advise, each expert's opinion carries less weight in the decision-making process. ${ }^{11}$ To see this, suppose that expert 1 observes $\hat{\theta}_{1}=0$ but claims $m_{1}=1$. In this case, the decision maker chooses $a_{2}=1$ if and only if $m_{2}=1$. Given that expert 2 adopts the truth-telling strategy, however, this means $\hat{\theta}_{2}=1$, in which case

\footnotetext{
${ }^{11}$ The logic behind this result is discussed in greater detail in Ishida and Shimizu [13].
} 
the unbiased probability of $\theta=1$ is exactly one half. ${ }^{12}$ At this point, therefore, exert 1 has nothing to lose by recommending $m_{1}=1$. In contrast, when $\hat{\theta}_{2}=0$, expert 2 claims $m_{2}=0$ and the decision maker follows this advice by choosing $a_{2}=0$. From the viewpoint of expert 1 , his advice is ignored when it is more likely to be wrong (when $\hat{\theta}_{1}=\hat{\theta}_{2}=0$ ), which substantially lowers the cost of lying. Although it is ex post optimal to overrule expert 1 's advise, this capability makes full separation less feasible.

It is certainly true that amassing more information generally leads to a better choice when there are no incentive issues and truthful information can be solicited at no cost. This natural conclusion does not necessarily hold true, however, when information is disclosed strategically, i.e., there are indeed such things as "asking too many opinions" in strategic environments. We summarize this observation as follows (the proof omitted).

Proposition 2 There exists no fully separating truth-telling equilibrium if $2 p r(1-r) b>d$.

The proposition states that when the cost of waiting is relatively low, there is no way to induce truthful information from both of the agents. The important point here is that the decision maker can no longer obtain any useful information once she becomes (or is expected to become) as informed about the current state of nature as each expert: in the current setting, the decision maker can obtain useful information only from one expert because that will make her as informed. This logic thus applies to a more general $T$-period setting in which the decision maker can solicit opinions from at most $T>2$ experts. To see this, consider a $T$-period version of the model where $p_{t}=0$ for $t=1,2, \ldots, T-1$ and $p_{T}=p$. In this extended setup, the condition for truth telling cannot be satisfied for any expert $t^{\prime}, t^{\prime}=1,2, \ldots, T-1$ if expert $T$ adopts the truth-telling strategy while all the other experts lie.

It is worth noting here that in the static setting, Assumption 1 is sufficient to assure the existence of fully separating equilibria. One interesting fact about this result is that in the current dynamic setting, the existence of fully separating equilibria hinges on such parameters as $d$ (which measures how costly it is to wait) and $p$ (which measures how strategic the underlying environment is), which would normally have no impact on the feasibility of truthful communication. The proposition shows that full separation cannot be attained in equilibrium if the cost of soliciting a second opinion is too small ( $d$ is too small), or if the likelihood of obtaining useful information on her own is too large ( $p$ is too large). This result offers several interesting implications which we discuss later in section 4 .

\footnotetext{
${ }^{12}$ This is the case because we assume the symmetric information structure where the two states are equally likely ex ante and the two signals are equally informative. This assumption is obviously made to simplify the argument and make our point more emphatically, as our logic holds even when we allow for some degree of asymmetries in the information structure.
} 
Given this impossibility result, we now seek for equilibria where the decision maker gains some useful information when she is not decisive enough. To this end, it is useful to note that there is no equilibrium where no expert (with dissonant preferences) communicates truthfully under Assumption 1 either, because if one expert lies, there is always an incentive for the other to communicate truthfully. ${ }^{13}$ This eventually leaves us with two possibilities, which we label as PIE-1 and PIE-2 for clarity (PIE for "partially informative equilibrium"), when the decision maker asks for a second opinion.

PIE-1: Expert 1 always communicates truthfully while expert 2 always claims $m_{2}=1$ when he is called upon to make advice. ${ }^{14}$ The decision maker asks for a second opinion when $m_{1}=1$.

PIE-2: Expert 2 always communicates truthfully when he is called to make advice while expert 1 always claims $m_{1}=1$. The decision maker always asks for a second opinion.

Proposition 3 (i) PIE-1 exists if $(1-p)(r-B) \geq p r(1-r) b$ and $2 p r(1-r) b>d$. (ii) PIE-2 exists if $r-B>d$.

Proof: See Appendix.

Some remarks are in order. First, among the two possibilities, PIE-2 is far more likely than PIE-1. To see this, note that $r-B>d$ implies $2 p r(1-r) b>d$, so that whenever the fully separating equilibrium fails to exist, there always exists PIE-2. This also indicates that there exists a range $D:=[2 p r(1-r), r-B)$ such that multiple equilibria exist for $d \in D$. The intuition for the emergence of multiple equilibria is as follows. On one hand, as we have seen, the incentive to communicate truthfully diminishes when the decision maker is more likely to obtain a second opinion. On the other hand, the incentive to ask for a second opinion is stronger when expert 1's information is less reliable than what she can expect to gain in period 2. This relationship thus suggests a possibility that the decision maker's (possibly irrelevant) personal characteristics or reputations may make a difference: when $d \in D$, the decision maker may not be able to achieve full separation when the expert believes that she is not decisive enough to take an action immediately, which in turn makes her indeed less decisive in a self-fulfilling way. We will explore more on this point later in section 4.

To summarize, Figure 1 illustrates the region under which each type of equilibrium exists. Note that we restrict attention to the case where the bias $b$ is small relative to the accuracy

\footnotetext{
${ }^{13}$ See the proof of Proposition 3 for more details.

${ }^{14}$ Of course, in this equilibrium, there is no point in communicating with expert 2 once the decision maker has failed to observe any signal on her own, so she may not ask for advice anyway.
} 
of information $r$, so that communication is supposed to be easy. Even under this restriction, one can see that partially informative equilibria exist for a fairly wide range of the parameter space.

\section{[figure 1]}

\subsection{The virtue of decisiveness}

We have thus far seen three different types of equilibrium: fully separating, PIE-1, and PIE2. In partially informative equilibria such as PIE-1 and PIE-2, there is always some type of expert who would not communicate truthfully, and the decision maker hence needs more time to solicit useful information. This possibility can be eliminated altogether, however, if the decision maker can somehow commit to the one-and-done strategy under which expert 1, the only expert to make advise, always has an incentive to communicate truthfully. Here, we suppose that such a commitment is indeed feasible, e.g., by intentionally raising the cost of waiting $d$, and explore when it is desirable for the decision maker to give up the discretion to ask for a second opinion by committing to the one-and-done strategy.

Proposition 4 The commitment to the one-and-done strategy weakly increases the decision maker's expected payoff if one of the following conditions is satisfied: (i) $d \geq 2 \operatorname{pr}(1-r) b$ or (ii) $2 p r(1-r) b \geq d$ and $\operatorname{pr}(1-r) b>(1-p)(r-B)$.

Proof: See Appendix.

The proposition shows that the commitment to the one-and-done strategy is indeed beneficial for the decision maker under a wide range of circumstances. ${ }^{15}$ What is particularly intriguing is the fact that the commitment is always desirable when $d \in D$ and both the fully separating equilibrium and PIE-2 exist. When there exist multiple equilibria, the actual outcome may depend on intrinsically irrelevant factors such as the decision maker's reputation as a decisive leader or her trustfulness. For instance, when the decision maker is perceived as indecisive for some reason, the expert is less willing to communicate truthfully, which in turn justifies the decision maker's decision to ask for a second opinion. On the other hand, when the decision maker does not trust her expert's ulterior motive, she is effectively forced to ask for a second opinion, which in turn makes the expert less willing to communicate

\footnotetext{
${ }^{15}$ Note that the value of the commitment is evaluated only from the viewpoint of the decision maker, where the cost of waiting is given by $d$. A delay to take an action can be costly for the organization in many regards, however, which include the costs that are not necessarily internalized by the decision maker. One can thus argue that when a delay yields an additional external cost to the organization, the commitment to the one-and-done strategy is even more valuable from the viewpoint of the organization as a whole.
} 
truthfully. In either case, as the proposition implies, the organization is trapped in the less efficient equilibrium, i.e., PIE-2, where the experts withhold useful information until the end. The commitment to the one-and-done strategy, if it is feasible at all, is quite effective in putting an end to this vicious circle, by keeping expert 1's incentives straight and making the underlying environment less strategic.

\section{Discussion}

\subsection{Interpretations}

The analysis presented above suggests that full separation is feasible only when the decision maker adopts the one-and-done strategy. We say, mostly for expositional clarity, that the decision maker is decisive when she can do so in a credible manner. It is up to interpretation, though, what actually makes a decision maker "decisive" in the current context, as being decisive in this model merely refers to a superficial behavioral characteristic which endogenously arises in the model. Here, we offer several interpretations of our main result by relating this behavioral characteristic to the primitives of the model.

Biased preferences hurt: As can be seen from Proposition 1, an increase in $b$ makes truthful communication less likely. This means that the presence of the preference bias limits the decision maker's ability to extract information from experts. Of course, the presence of the preference bias is almost always an obstacle to efficient information transmission: in a canonical model of cheap talk a la Crawford and Sobel [5], only the babbling equilibrium survives when the preference bias exceeds some threshold. ${ }^{16}$ In this context, however, the presence of the preference bias impedes efficient communication in a little different way. Here, a biased decision maker cannot extract truthful information from experts because they know that the decision maker is not open to the unfavorable opinion: whenever the decision maker receives a bad news, she goes on to ask for a second opinion, rather than to take an action recommended by the expert. The lack of commitment to abide by the first opinion, however, diminishes the first expert's to incentive to report truthfully because his opinion weighs less in the decision-making process when the decision maker solicits multiple opinions. This result hence suggests that an effective leader is the one who is fair and open-minded enough to accept any opinions even when they are in direct conflict with her own personal interests.

Friendly environments may hurt: The driving force of the model is the fact that the more informed the decision maker is, the less information she can extract from each expert

\footnotetext{
${ }^{16}$ Similarly, in the current model, communication becomes totally meaningless when the bias is sufficiently large, because the decision maker simply chooses her preferred action regardless of the messages.
} 
via communication. The decision maker is less tempted to ask for a second opinion when it is less likely to obtain any useful information by waiting. This could actually benefit her because that works as a commitment device. In contrast, when the decision maker is more likely to come across useful information by chance, through the exogenous (partial) resolution of uncertainty, the temptation might be just too large and simply irresistable. One can thus argue that the decision maker's decisiveness is not only determined by her own personal characteristics, but also by the underlying environment in which the decision is to be made.

Too many experts around?: Another environmental factor which could make a difference is the cost of waiting, which is captured by $d$ in this setup. It is obvious that the decision maker is tempted to ask for a second opinion when $d$ is close to zero and the cost of waiting is relatively low. An obvious and straightforward interpretation of this fact is that the decision maker tends to be indecisive when she is relatively patient, which lowers the relative value of taking an action today. Alternatively, the cost of waiting also depends on how frequently the decision maker can an expert to solicit opinions from. According to this latter interpretation, it is not necessarily better to have access to multiple information sources, as it may only impede efficient communication. As we will discuss next, this fact yields some critical implication when the distribution of information is to some extent under the decision maker's control.

Career concerns help: Suppose that the decision maker has a career concern and has an incentive to establish her reputations. Suppose also that outside parties can observe at what timing the decision maker has made the decision. In this case, there arises an additional benefit of taking an action in period 1 , as it signals that she is more likely to be decisive. Let this benefit be $v$. The total payoff for the decision maker is then

$$
u^{d}(\theta, a, t)=\mathbb{I}(\theta=a)+b \mathbb{I}(a=1)+v(2-t)-d(t-1) .
$$

Using this modified specification, the condition for full separation is given by

$$
d+v \geq 2 \operatorname{pr}(1-r) b
$$

It is then clear from this that an increase in $v$ makes full separation more likely. To see the full impact of career concerns, we obviously need to see how $v$ is determined in equilibrium. Aside from this, however, the presence of career concerns gives the decision maker an additional reason to take an action immediately, which works as a credible commitment to induce truthful information. 


\subsection{Implications}

The main reason why communication cannot be made fully informative in the current setup is the lack of commitment, on the part of the decision maker, not to ask for a second opinion. In other words, the efficiency of communication can be restored if the decision maker can put herself in an environment where it is simply not feasible or optimal to ask for a second opinion. Here, we briefly discuss ways in which to raise the cost of obtaining a second opinion in organizations so that the decision maker can credibly be decisive.

One way to do so is to set a deadline for the project, which might make it harder to find another expert to solicit an opinion from in time. To put this possibility into the current context, the difficulty for the decision maker arises precisely because she faces a dynamic problem which gives her an opportunity to solicit two opinions. Now suppose that the decision maker sets a deadline for the project and makes it a one-period static problem in a credible way. If this is feasible at all, as we have seen, there exists a fully separating equilibrium under Assumption 1, irrespective of such parameters as $d$ and $p$ : full information can be extracted no matter how indecisive the decision maker may be.

The analysis also suggests that communication becomes increasingly difficult in strategic environments when information is dispersed sparsely among different sources. This means that if the decision maker can reasonably control the distribution of information, it might be optimal to limit the number of information sources, e.g., by delegating or specializing information acquisition to a single party. The tradeoff is clear: on one hand, when each expert is endowed only with imperfect information, there is a benefit of aggregating information from different sources to make a better forecast; on the other hand, as we have shown, the existence of multiple information sources can be an impediment to efficient communication in strategic environments. When the cost outweighs the benefit, it is optimal to delegate information acquisition to a single source and rely on it exclusively; when the benefit outweighs the cost, it is optimal to diversify information sources and aggregate them via communication. The deciding factor is the strategic nature of communication, namely how severe the conflict of interests is between the decision maker and each expert, which provides an implication for how responsibility regarding information acquisition should be allocated in organizations. When the conflict of interests is fairly severe and communication is highly strategic in nature, more emphasis should be placed on facilitating communication, meaning that information acquisition should be more specialized. ${ }^{17}$ When there is no conflict of interests and communication is nonstrategic, information sources should be more diversified to complement each other.

\footnotetext{
${ }^{17}$ This logic illuminates yet another virtue of initiative, in a way different from Aghion and Tirole [1] and Zabojinik [18].
} 


\section{Extension: a model of private consultation}

We have thus far assumed that each expert knows whether he is the first or second one to make advice. In some cases, however, it is also plausible to assume that each expert does not know how many opinions the decision maker has solicited in the past. This is more likely to be the case when communication between the decision maker and each expert is done in private, which might be labeled as the case of private consultation. In this section, we briefly consider this case to show that our insight generally applies, perhaps in a manner even more emphatic.

To this end, we make two modifications to the basic setup. First and foremost, we assume that each expert does not know whether he is the first or second one to make advice. ${ }^{18}$ Second, while we assume that the decision maker may obtain information on her own in the model of public consultation, this possibility is not necessary in the model of private consultation. ${ }^{19}$ We hence assume $p=0$, i.e., the decision maker must always rely on the experts to make the model symmetric between the two periods. We then seek for conditions under which there exist fully separating truth-telling equilibria in which every expert who is called upon to make advice chooses $m_{t}=\hat{\theta}_{t}$.

Again, since the condition for truth telling is naturally more stringent when $\hat{\theta}_{t}=0$, we focus on that case. First, as above, if the decision maker adopts the one-and-done strategy, the problem is exactly identical to that in the model of public consultation: the fact that $t$ is not observable to each expert has no bite since this is virtually a static problem with only one expert, and the expert who is called upon to make advice knows that he must be the first one. If the decision maker asks for a second opinion when $m_{1}=1$, on the other hand, truth telling cannot be induced on the equilibrium path. To see this, consider an expert who is called upon to make advice. Let $q$ denote the expert's belief that he is the first one to make advice. There are two cases we need to examine.

$t=1$ : If $m_{1}=0$, then $a_{1}=0$ which yields the expected payoff of $r$. If $m_{1}=1$, the decision maker asks for a second opinion. The expected payoff is $r+2 r(1-r) b$.

$t=2$ : The fact that the decision maker asks for a second opinion means that $m_{1}=\hat{\theta}_{1}=1$. If $m_{2}=0$, then $a_{2}=0$ and the expected payoff is $1 / 2$; if $m_{2}=1$, then the expected payoff is $1 / 2+b$.

\footnotetext{
${ }^{18}$ This also necessarily means that each expert cannot observe the message sent by the other expert.

${ }^{19}$ This possibility is critical in the model of public consultation, because the decision maker can always gain useful information with positive probability.
} 
It follows from this that each expert has an incentive to communicate truthfully only if

$$
q r+\frac{1-q}{2} \geq q[r+2 r(1-r) b]+\frac{(1-q)(1+2 b)}{2},
$$

which is clearly never satisfied for any belief $q<1$ as long as $b>0$, no matter how small it is.

This means that full separation is not feasible if the decision maker cannot resist the temptation to ask for a second opinion, given that each expert adopts the truth-telling strategy. Under Assumption 1, it is optimal to choose $a_{1}=1$, if not $a_{1}=W$, when $m_{1}=1$. The expected payoff in this case is then $r$. If the decision maker chooses to ask for a second opinion, on the other hand, the expected payoff is $r+2 r(1-r) b-d$. It is optimal for the decision maker to adopt the one-and-done strategy if

$$
d \geq 2 r(1-r) b
$$

In other words, there exists no fully separating equilibrium when (3) fails to hold. We summarize this finding below (the proof omitted).

Proposition 5 In the case of private consultation, there exists no fully separating truthtelling equilibrium if $2 r(1-r) b>d$.

\section{Conclusion}

This paper provides a simple model of strategic communication where the decision maker solicits opinions sequentially from imperfectly informed experts. The entire argument hinges on the observation that communication cannot be made fully informative when there are too many information sources, which holds in a wide variety of circumstances which involve strategic information transmission. Building on this logic, we show that the decision maker can induce truthful information from the expert only when she can resist the temptation to ask for a second opinion and get right to the task. This result illuminates a virtue of being decisive in strategic environments, especially why it is often better to do the wrong thing rather than to do nothing.

\section{References}

[1] Philippe Aghion and Jean Tirole. Formal and real authority in organizations. Journal of Political Economy, 105(1):1-29, 1997.

[2] David Austen-Smith. Interested experts and policy advice: Multiple referrals under open rule. Games and Economic Behavior, 5(1):3-43, 1993. 
[3] Patrick Bolton, Markus K. Brunnermeier, and Laura Veldkamp. Leadership, coordination and mission-driven management. mimeo, 2009.

[4] Ying Chen. Communication with two-sided asymmetric information. mimeo, 2009.

[5] Vincent P. Crawford and Joel Sobel. Strategic information transmission. Econometrica, 50(6):1431-1451, 1982.

[6] Ines Moreno de Barreda. Cheap talk with two-sided private information. mimeo, 2010.

[7] Eric Van den Steen. Organizational beliefs and managerial vision. Journal of Law, Economics, and Organization, 21(1):256-283, 2005.

[8] Torun Dewan and David P. Myatt. Leading the party: Coordination, direction, and communication. American Political Science Review, 101(4):825-843, 2007.

[9] Torun Dewan and David P. Myatt. The qualities of leadership: Direction, communication, and obfuscation. American Political Science Review, 102(3):351-368, 2008.

[10] Torun Dewan and David P. Myatt. On the rhetorical strategies of leaders: Speaking clearly, standing back, and stepping down. mimeo, 2009.

[11] Andrea Galeotti, Christian Ghiglino, and Francesco Squintani. Strategic information transmission in networks. Discussion Paper Series No. 668, University of Essex, 2009.

[12] Benjamin E. Hermalin. Toward an economic theory of leadership: Leading by example. American Economic Review, 88(5):1188-1206, 1998.

[13] Junichiro Ishida and Takashi Shimizu. Cheap talk with an informed receiver. mimeo, 2010.

[14] Ernest K. Lai. Expert advice for amateurs. mimeo, 2009.

[15] John Morgan and Phillip C. Stocken. Informal aggregation in polls. American Economic Review, 98(3):864-896, 2008.

[16] Julio J. Rotemberg and Garth Saloner. Benefits of narrow business strategies. American Economic Review, 84(5):1330-1349, 1994.

[17] Julio J. Rotemberg and Garth Saloner. Visionaries, managers, and strategic direction. RAND Journal of Economics, 31(4):693-716, 2000.

[18] Jan Zabojnik. Centralized and decentralized decision making in organizations. Journal of Labor Economics, 20(1):1-22, 2002. 


\section{Appendix}

Proof of Proposition 1: Let $\sigma_{1}\left(m_{1}, m_{2}\right):=\operatorname{Pr}\left(\theta=1 \mid m_{1}, m_{2}\right)$ denote the decision maker's posterior belief when she solicits advice from both of the experts. We first show that there is no fully separating equilibrium in period 2, given that expert 1 adopts the truth-telling strategy. Suppose, on the contrary, that expert 2 also adopts the truth-telling strategy. The posterior beliefs are then given by

$$
\sigma_{2}(1,0)=\frac{1}{2}, \sigma_{2}(1,1)=\frac{r^{2}}{r^{2}+(1-r)^{2}}
$$

Expert 2's message is pivotal because $\sigma_{2}(1,1)>B>\sigma_{2}(1,0)$. If the expert communicates truthfully when $\hat{\theta}=0$, the expected payoff is $1 / 2$; if he lies, it is $1 / 2+b$. This means that the incentive compatibility constraint cannot be satisfied for any $b$, no matter how small it is.

Given that expert 2 does not communicate truthfully, the decision maker can obtain useful information only with probability $p$ : in this case, she observes $\hat{\theta}_{2}=1$ and chooses $a_{2}=1$ with probability $r$ while she observes $\hat{\theta}_{2}=0$ and chooses $a_{2}=0$ with the remaining probability. The decision maker then takes an action immediately when $m_{1}=1$ if

$$
r \geq p\left[r^{2}+r(1-r)(1+2 b)\right]+(1-p) r-d,
$$

which is reduced to $d \geq 2 \operatorname{pr}(1-r) b$.

Proof of Proposition 3: We know that $a_{1}=0$ when $m_{1}=0$ and $a_{2}=0$ if $m_{1}=1$ and $m_{2}=0$. Define $\ell_{t}$ as the probability that expert $t$ sends $m_{t}=1$ when $\hat{\theta}_{t}=0$ and he is called upon to make advice (for the case of expert 2). ${ }^{20}$ Given the experts' strategies, one can show that

$$
\begin{gathered}
\sigma_{2}(0,0)=\frac{(1-r)^{2}}{r^{2}+(1-r)^{2}}, \sigma_{2}(0,1)=\frac{r(1-r)+(1-r)^{2} \ell_{2}}{2 r(1-r)+\left[r^{2}+(1-r)^{2}\right] \ell_{2}}, \\
\sigma_{2}(1,0)=\frac{s\left(\ell_{1}\right)(1-r)}{s\left(\ell_{1}\right)(1-r)+\left[1-s\left(\ell_{1}\right)\right] r}, \\
\sigma_{2}(1,1)=S\left(\ell_{1}, \ell_{2}\right):=\frac{s\left(\ell_{1}\right)\left[r+(1-r) \ell_{2}\right]}{s\left(\ell_{1}\right)\left[r+(1-r) \ell_{2}\right]+\left[1-s\left(\ell_{1}\right)\right]\left[1-r\left(1-\ell_{2}\right)\right]} \\
=\frac{\left[r+(1-r) \ell_{1}\right]\left[r+(1-r) \ell_{2}\right]}{\left[r+(1-r) \ell_{1}\right]\left[r+(1-r) \ell_{2}\right]+\left[1-r\left(1-\ell_{1}\right)\right]\left[1-r\left(1-\ell_{2}\right)\right]} .
\end{gathered}
$$

\footnotetext{
${ }^{20}$ As noted earlier, there exists an uninformative (babbling) equilibrium where both experts lie and the decision maker chooses $a_{1}=0$. Here, we do not consider this possibility and focus on the case where the decision maker asks for a second opinion when $m_{1}=1$.
} 
where

$$
s\left(\ell_{1}\right):=\frac{r+(1-r) \ell_{1}}{1+\ell_{1}}
$$

We have already seen that if one expert communicates truthfully, the incentive compatibility condition can never be satisfied for the other expert. This means that there are three possible pure-strategy equilibria within the class of equilibria where the decision maker asks for a second opinion when $m_{1}=1$ : (a) only expert 1 communicates truthfully; (b) only expert 2 communicates truthfully; and (c) neither communicates truthfully. Given $m_{1}=1$, the decision maker asks for a second opinion if

$$
\begin{aligned}
\max \left\{s\left(\ell_{1}\right), 1-s\left(\ell_{1}\right)+b\right\}< & p\left(r+\left\{s\left(\ell_{1}\right)(1-r)+r\left[1-s\left(\ell_{1}\right)\right]\right\} b\right) \\
& +(1-p)\left(s\left(\ell_{1}\right)\left[r+(1-r) \ell_{2}\right]+\left[1-s\left(\ell_{1}\right)\right]\left(1-\ell_{2}\right) r\right. \\
& \left.+\left(1-\ell_{2}\right)\left\{s\left(\ell_{1}\right)(1-r)+r\left[1-s\left(\ell_{1}\right)\right]\right\} b\right)-d .
\end{aligned}
$$

Only expert 1 communicates truthfully: The incentive compatibility condition for expert 2 can never be satisfied in this case. Given this and the decision maker asks for a second opinion when $m_{1}=1, \ell_{1}=0$ if

$$
r \geq p[r+2 r(1-r) b]+(1-p)(1-r+b),
$$

which can be written as

$$
(1-p)(2 r-1-b) \geq 2 \operatorname{pr}(1-r) b \Leftrightarrow(1-p)(r-B) \geq \operatorname{pr}(1-r) b .
$$

It also follows from (4) that the decision maker asks for a second opinion when $m_{1}=1$ iff $2 p r(1-r) b>d$.

Only expert 2 communicates truthfully: The incentive compatibility condition for expert 1 can never be satisfied in this case. Given this, $\ell_{2}=0$ if

$$
1-\frac{s(1)(1-r)}{s(1)(1-r)+r[1-s(1)]} \geq \frac{s(1)(1-r)}{s(1)(1-r)+r[1-s(1)]}+b,
$$

which can be written as

$$
\frac{r[1-s(1)]}{s(1)(1-r)+r[1-s(1)]}=r \geq B \text {. }
$$

It also follows from (4) that the decision maker asks for a second opinion when $m_{1}=1$ if

$$
r-B>d
$$


Neither communicates truthfully: It is easy to see that this type of equilibrium is not feasible under Assumption 1. To see this, note that if $\ell_{1}=1$, expert 2 also lies if (5) fails to hold. This possibility is, however, ruled out by Assumption 1.

Proof of Proposition 4: Let $\pi_{k}$ denote the expected payoff for the decision maker in PIE$k$; also, let $\pi_{F S}$ denote the expected payoff when she commits to the one-and-done strategy. One can then easily compute

$$
\begin{gathered}
\pi_{1}=\frac{r+b}{2}+\frac{r+2 p r(1-r) b-d}{2}=r+\frac{b}{2}+\frac{2 p r(1-r) b-d}{2}, \\
\pi_{2}=r+\frac{b}{2}-d, \\
\pi_{F S}=r+\frac{b}{2} .
\end{gathered}
$$

It is immediate to see that $\pi_{F S}>\pi_{2}$ for any $d>0$, meaning that PIE-2 always yields a lower payoff to the decision maker than the fully separating equilibrium. Note also that $\pi_{F S} \geq \pi_{1}$ if

$$
d \geq 2 \operatorname{pr}(1-r) b
$$

This suggests that PIE-1 yields the highest payoff in the range where it exists. For the remaining part, the fully separating equilibrium is the most desirable for the decision maker and hence the commitment strictly raises the decision maker's expected payoff. The two conditions, (i) and (ii), show the range where PIE-1 fails to exist. 


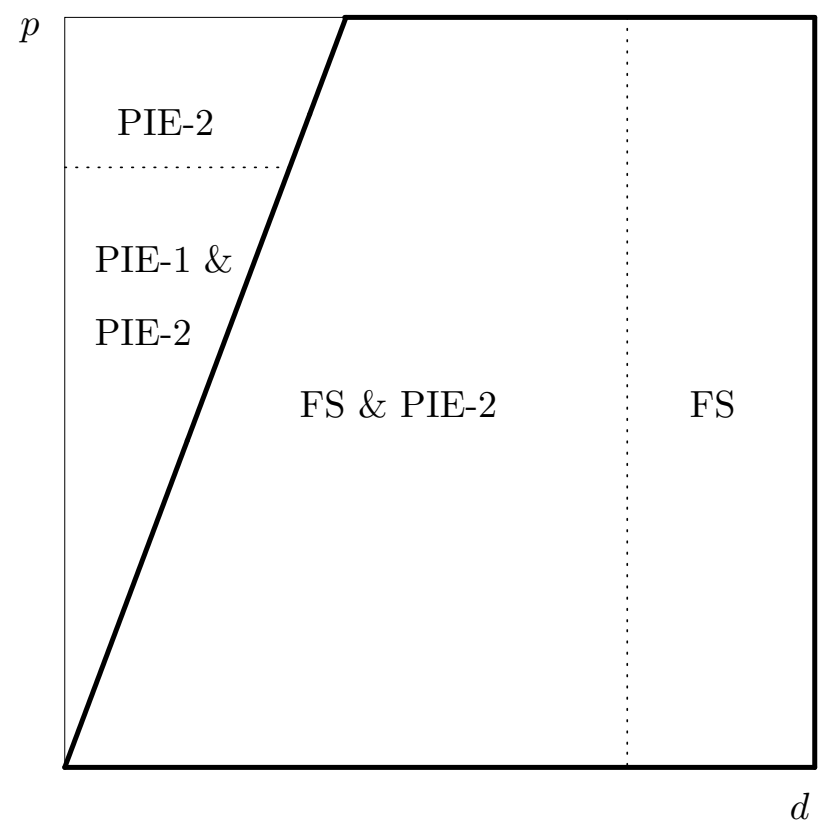

Figure 1: Equilibria when $b$ is small

Note: FS indicates the region in which the fully separating equilibrium exists 